\title{
Avaliação radiográfica da silhueta cardíaca pelo método vertebral heart size em cães da raça Yorkshire Terrier clinicamente normais
}

\author{
[Radiographic evaluation of the cardiac silhouette in clinically normal Yorkshire Terrier dogs through \\ the vertebral heart size method] \\ M.G. Castro ${ }^{1}$, R.C.S. Tôrres ${ }^{1}$, R.B. Araújo ${ }^{1}$, R.A.L. Muzzi ${ }^{2}$, E.F. Silva ${ }^{3}$ \\ ${ }^{1}$ Departamento de Clínica e Cirurgia Veterinárias - UFMG - Belo Horizonte, MG \\ ${ }^{2}$ Departamento de Medicina Veterinária - UFLA - Lavras, MG \\ ${ }^{3}$ Aluno de pós-graduação - Escola de Veterinária - UFMG - Belo Horizonte, MG
}

\begin{abstract}
RESUMO
Determinou-se valor médio do vertebral heart size (VHS) em cães da raça Yorkshire Terrier. Foram selecionados 30 cães clinicamente normais, com média de peso de $2,42 \pm 0,64 \mathrm{~kg}$ e idades entre um e seis anos. Os animais foram submetidos ao exame radiográfico do tórax nas projeções lateral direita, ventrodorsal e dorsoventral. Foram feitas mensurações para avaliação cardíaca e da profundidade e

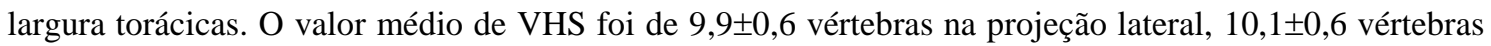
na projeção ventrodorsal e $10,0 \pm 0,6$ vértebras na projeção dorsoventral. Não houve diferença entre esses valores. Cinco animais (16,7\%) apresentaram VHS acima de 10,5 vértebras, valor sugerido como limite superior para a maioria das raças, em projeção lateral. Os valores de VHS correlacionaram-se com peso corporal nas projeções lateral e ventrodorsal. A razão profundidade:largura torácica apresentou valor médio de $0,75 \pm 0,06$. Não foi observada correlação entre a qualidade do tórax e o VHS.
\end{abstract}

Palavras-chave: cão, radiografia torácica, silhueta cardíaca, VHS

\begin{abstract}
The mean vertebral heart size (VHS) was established for Yorkshire Terrier dogs. Thirty clinically normal dogs with mean weight of $2.42 \pm 0.64 \mathrm{~kg}$ and ages varying from one to six years of age, were studied. The animals were submitted to right lateral, ventrodorsal and dorsoventral thoracic radiographs. The Buchanan e Bücheler method was applied to the cardiac silhouette and thoracic depth and width. The VHS was $9.9 \pm 0.6$ vertebrae on lateral, $10.1 \pm 0.6$ on ventrodorsal and 10.0 \pm 0.6 on dorsoventral radiographs. There was no difference among these values. Five animals (16.7\%) presented VHS values exceeding 10.5, the value suggested as upper limit for most breeds in lateral view. However, $95 \%$ of the animals in this study provide VHS values below the upper limit, and this should be equal to 11 vertebrae. The VHS values had significant correlation with body weight on lateral and VD radiographs. The mean depth:width ratio was $0.75 \pm 0.06$. There was no correlation among VHS and depth:width ratios.
\end{abstract}

Key words: dog, thoracic radiography, cardiac silhouette, VHS

\section{INTRODUÇÃO}

Diversos métodos foram desenvolvidos para avaliar a silhueta cardíaca de cães em radiografias torácicas. $\mathrm{O}$ método radiográfico mais utilizado para avaliação cardíaca é o qualitativo ou subjetivo, pelo qual o profissional faz uso de seus conhecimentos sobre anatomia radiográfica, a fim de detectar alterações de forma e tamanho de partes específicas do coração. As vantagens desse método são basicamente a conveniência e a rapidez, porém deve ser adotado por radiologistas experientes, que conheçam as variações normais que a imagem pode apresentar (Lamb e Boswood, 2002; Hansson, 2004; Soares et al., 2004).

Recebido em 14 de janeiro de 2011

Aceito em 27 de junho de 2011

E-mail: michellegc@ig.com.br 
As variações na aparência da silhueta cardíaca podem ocorrer devido a vários fatores, incluindo posicionamento do animal, projeção radiográfica, fase da respiração, estado corporal do animal, conformação torácica e fase do ciclo cardíaco. Sendo assim, a interpretação de radiografias torácicas deve ser realizada com cautela para evitar diagnósticos incorretos de doença cardíaca (Hansson, 2004; Kealy e McAllister, 2005).

Dentre os métodos radiográficos propostos, os de mensuração visam avaliar de forma quantitativa o tamanho do coração, com a finalidade de diminuir a subjetividade da avaliação qualitativa (Buchanan e Bücheler, 1995; Litster e Buchanan, 2000). A proposta mais objetiva para avaliar o tamanho do coração é o método denominado vertebral heart size (VHS), desenvolvido por Buchanan e Bücheler (1995). Utilizando o sistema de unidade vertebral, o método compara as dimensões cardíacas com o comprimento das vértebras torácicas, de forma a se determinar o VHS, ou seja, o número que representa o tamanho do coração em relação ao tamanho do animal (Bavegems et al., 2005; Ghadiri et al., 2008).

Os valores médios de VHS obtidos por

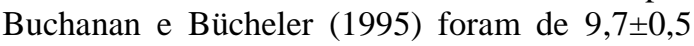
vértebras (média \pm desvio-padrão) em projeção lateral, 10,2 $\pm 0,8$ vértebras em projeção ventrodorsal e 10,2 $\pm 1,5$ vértebras em projeção dorsoventral para cães de várias raças. Como 98\% das radiografias laterais apresentaram VHS menor ou igual a 10,5 vértebras, este valor foi sugerido como limite superior para um tamanho normal de silhueta cardíaca na maioria das raças. Todavia, em cães com tórax curto, como os da raça Schnauzer Miniatura, os autores sugerem como normal um valor de VHS de 11 vértebras. Por outro lado, para aqueles cães com tórax longo, como os Dachshunds, considera-se como normal o valor limite de 9,5 vértebras. Esses autores consideram ainda que o método de mensuração VHS tem uma grande utilidade na determinação de cardiomegalias em cães com alterações radiográficas mínimas, assim como permite a avaliação sequencial das radiografias de pacientes cardiopatas de forma mais precisa (Lamb et al., 2001; Nakayama et al., 2001; Pinto e Iwasaki, 2004).

Vários autores têm estudado e utilizado o VHS em suas pesquisas, em que foram observadas diferenças significativas entre os valores médios para as diferentes raças de cães, indicando a necessidade de padronizar os valores de VHS de acordo com a raça e a conformação torácica do animal. Foi demonstrado que cães das raças Boxer, Labrador Retriever, Cavalier King Charles Spaniel, Doberman Pinsher, Whippet e Greyhound apresentam valores de VHS acima da média indicada por Buchanan e Bücheler (1995) (Lamb et al., 2001; Bavegems et al., 2005; Marin et al., 2007).

Os animais da raça Yorkshire Terrier são frequentemente acometidos por cardiopatias congênitas, como a persistência do ducto arterioso, e por afecções adquiridas, como a endocardiose da valva mitral (Lamb et al., 2001; Castro et al., 2009). Além disso, essa raça é considerada como uma das mais predispostas ao colapso de traqueia (McReady et al., 2007; Ferian, 2009), afecção respiratória crônica que, além de apresentar sinais clínicos em comum com algumas cardiopatias, possibilita $\mathrm{o}$ desenvolvimento de importantes alterações no sistema cardiovascular (Marolf et al., 2007), sendo que o exame radiográfico do tórax permite o diagnóstico diferencial entre afecções respiratórias e cardíacas (Lamb e Boswood, 2002; Kealy e McAllister, 2005).

Pela grande variabilidade racial dos valores de VHS e pela escassez de estudos visando à avaliação cardíaca de cães hígidos da raça Yorkshire Terrier, o objetivo deste trabalho é aplicar o método proposto por Buchanan e Bücheler (1995), a fim de determinar o valor médio de VHS para esta raça.

\section{MATERIAL E MÉTODOS}

Foram utilizados 30 cães da raça Yorkshire Terrier clinicamente normais, sendo 14 machos e 16 fêmeas, com peso inferior a $4 \mathrm{~kg}$ e idades entre um e seis anos, de origem variada. Os cães foram selecionados por meio de exames clínico e eletrocardiográfico, além de avaliação laboratorial, que incluía a realização de hemograma completo, dosagem sérica de ureia, creatinina, alanina aminotransferase, aspartato aminotransferase, bilirrubinas, colesterol total, fosfatase alcalina, gama glutamil transpeptidase, proteínas totais e albumina. Os considerados hígidos foram submetidos ao exame radiográfico do tórax. 
Os animais foram posicionados em decúbito lateral direito, para projeção lateral direita, em decúbito dorsal, para projeção ventrodorsal, e em decúbito esternal, para projeção dorsoventral. Os exames radiográficos foram realizados na fase de inspiração máxima, porém radiografias laterais expiratórias também foram utilizadas para avaliar a ocorrência de colapso traqueal. A avaliação qualitativa das radiografias foi realizada a fim de excluir os animais que apresentassem quaisquer alterações radiográficas. Em seguida, as radiografias foram avaliadas quantitativamente, pelo método de mensuração VHS. Sendo assim, nas radiografias laterais, o eixo maior do coração foi medido com auxílio de um compasso e uma régua desde a borda ventral do brônquio principal esquerdo até o ponto mais distante do contorno ventral do ápice cardíaco. O compasso foi, então, reposicionado ao longo da coluna vertebral, iniciando na borda cranial da quarta vértebra torácica (T4). A distância correspondente ao eixo maior do coração foi transformada em valores com unidade de vértebras e aproximação de 0.1 vértebras. Com isso, o comprimento do coração foi registrado como o número de vértebras caudais àquele ponto. Para a mensuração do eixo menor, uma linha perpendicular ao eixo maior foi traçada na altura do terço central do coração. Em seguida, o eixo menor foi obtido da mesma maneira, começando a partir da borda cranial de T4. O comprimento e a largura do coração foram, então, somados para se obter o VHS (Fig. 1A). Nas radiografias ventrodorsais e dorsoventrais, os eixos maior e menor do coração foram determinados de modo semelhante ao descrito anteriormente, e as medidas obtidas foram reposicionadas nas projeções laterais, a partir da borda cranial de T4 (Fig.1B).
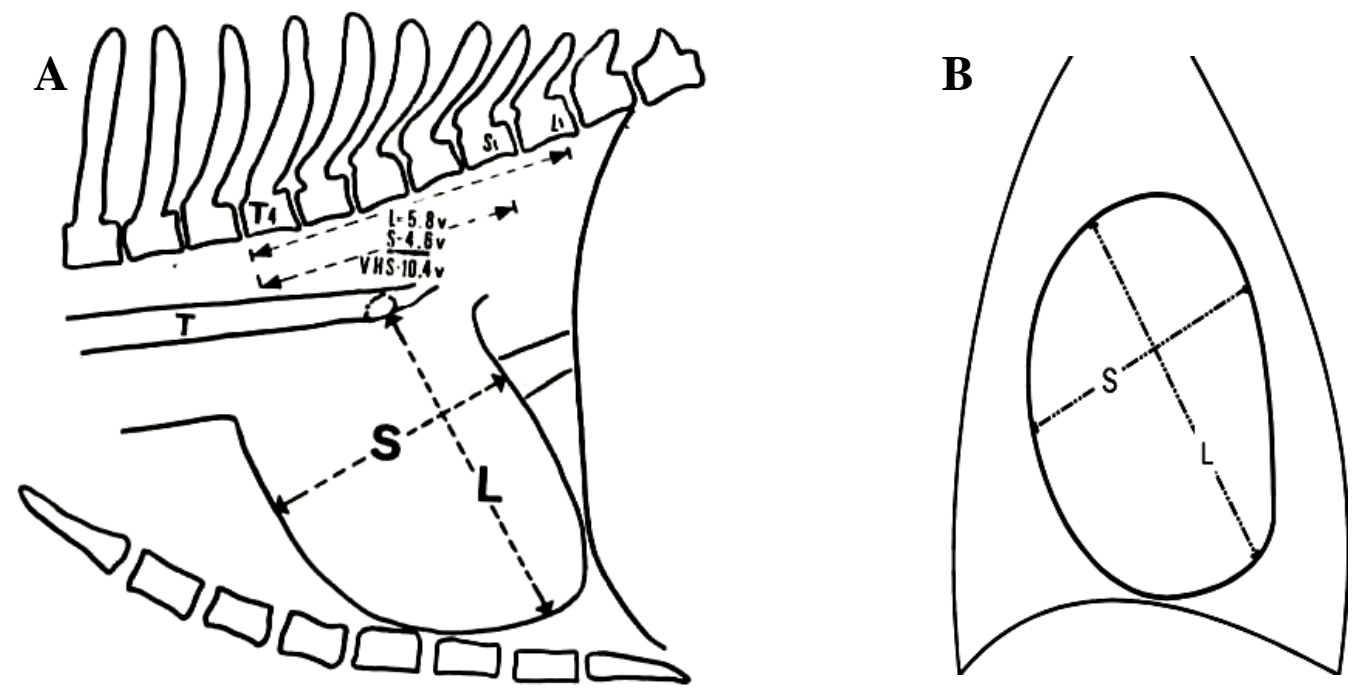

Fonte: Buchanan e Bücheler, 1995.

Figura 1. Cadela. Método de mensuração vertebral heart size (VHS) nas projeções lateral (A) e ventrodorsal ou dorsoventral (B). L, eixo maior; S, eixo menor; T, traqueia; T4, quarta vértebra torácica; $\mathrm{V}$, vértebras.

Para obtenção da qualidade do tórax - profundo, intermediário ou largo -, foram realizadas mensurações da profundidade e largura torácicas, seguindo as recomendações de Buchanan e Bücheler (1995). A profundidade do tórax (Pt) foi mensurada na projeção lateral e obtida a partir da distância entre a borda cranial do processo xifoide e a borda ventral da coluna vertebral, definida por uma linha perpendicular à coluna (Fig. 2A). A largura do tórax (Lt) foi mensurada na projeção ventrodorsal e/ou dorsoventral, e obtida a partir da distância entre as bordas mediais das oitavas costelas, junto às suas curvaturas mais laterais (Fig. 2B). De acordo com esses autores, o tórax canino é classificado como profundo se a relação Pt:Lt apresentar valor maior ou igual a 1,25, e largo se essa relação for menor ou igual a 0,75 . 

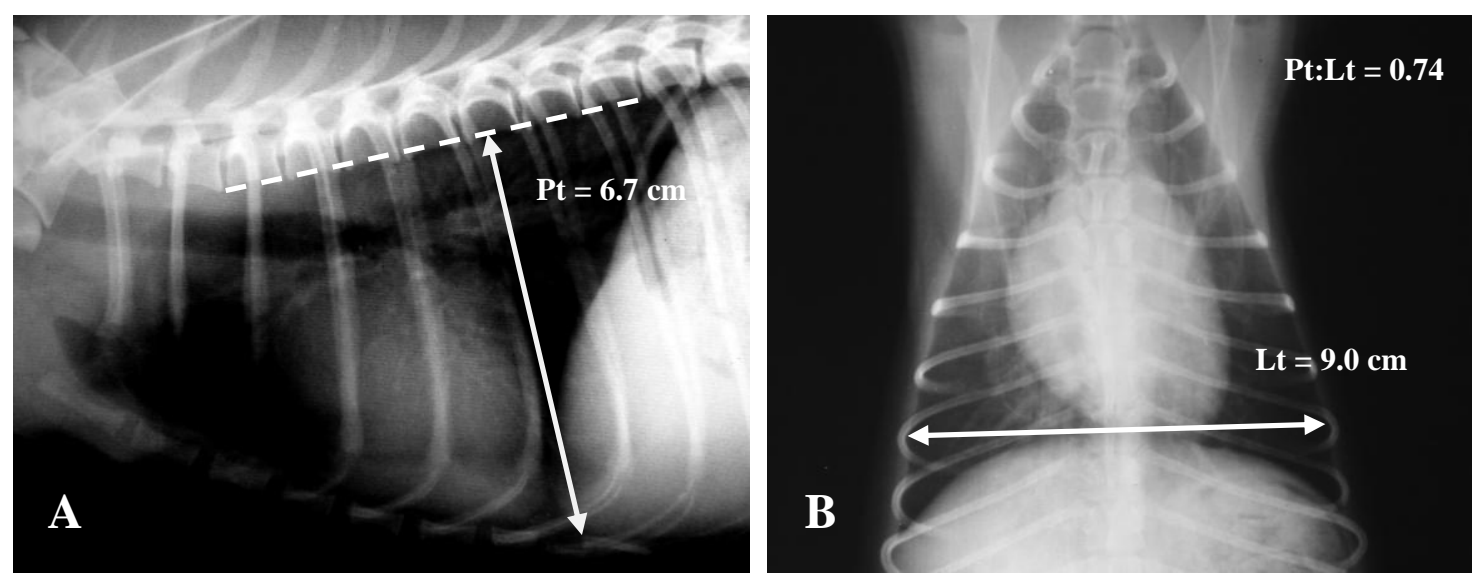

Figura 2. Método de mensuração da profundidade $(\mathrm{Pt})$ e largura torácicas $(\mathrm{Lt})$ para obtenção da qualidade do tórax em cadela da raça Yorkshire Terrier. A: Projeção lateral direita. B: Projeção ventrodorsal. Notase que a relação Pt:Lt obtida é de 0,74; sendo assim, esse animal apresenta tórax largo.

Os valores de VHS obtidos foram submetidos à análise estatística descritiva para o cálculo do valor médio e do desvio-padrão. Foi realizada, ainda, uma comparação entre os valores de VHS nas radiografias lateral, ventrodorsal e dorsoventral. O coeficiente de correlação de Pearson (r) foi calculado com a finalidade de estimar a correlação entre peso corporal e idade com os valores de VHS, assim como entre a qualidade do tórax e os valores de VHS. Considerou-se correlação positiva e significativa quando o coeficiente de correlação foi maior ou igual a 0,40 e a significância (P) menor ou igual a 0,05 . Em relação ao sexo, foi utilizado o teste $t$ para comparação entre machos e fêmeas (Sampaio, 2002).

\section{RESULTADOS E DISCUSSÃO}

A faixa etária variou de um a seis anos de idade, com média de 2,74 $\pm 1,24$ anos, e o peso de 1,5 a $4,0 \mathrm{~kg}$, com média de $2,42 \pm 0,64 \mathrm{~kg}$. Os machos pesaram em média $2,43 \pm 0,67 \mathrm{~kg}$, e as fêmeas $2,42 \pm 0,65 \mathrm{~kg}$. A amostra constituiu-se de $53 \%$ de fêmeas e $47 \%$ de machos.

$\mathrm{Na}$ avaliação qualitativa do tórax, não foram observadas alterações radiográficas significativas. A traqueia apresentou diâmetro normal em toda sua extensão, independentemente da fase respiratória. A silhueta cardíaca, o parênquima pulmonar e as outras estruturas torácicas apresentaram imagens radiográficas aparentemente normais.

$\mathrm{Na}$ avaliação quantitativa da silhueta cardíaca, o valor médio do VHS foi de 9,9 $\pm 0,6$ vértebras na projeção lateral (Fig. 3), sendo o intervalo de confiança de 8,7 a 11,1 vértebras (Fig. 4). Cinco animais $(16,7 \%)$ apresentaram VHS acima de 10,5 vértebras, valor sugerido por Buchanan e Bücheler (1995) como limite superior para a maioria das raças. Entretanto, para que $95 \%$ dos animais deste estudo apresentassem valores de VHS abaixo do limite superior, este deveria ser igual a 11 vértebras. A variação entre raças dos valores de VHS na projeção lateral já foi descrita (Lamb et al., 2001; Pinto e Iwasaki, 2004; Marin et al., 2007), confirmada pelos resultados deste estudo na raça Yorkshire Terrier. A determinação do VHS específico para cada raça, associada à avaliação qualitativa, é essencial para uma interpretação radiográfica adequada, evitando diagnóstico incorreto de cardiomegalia. A distribuição dos valores de VHS encontra-se na Fig. 5. 


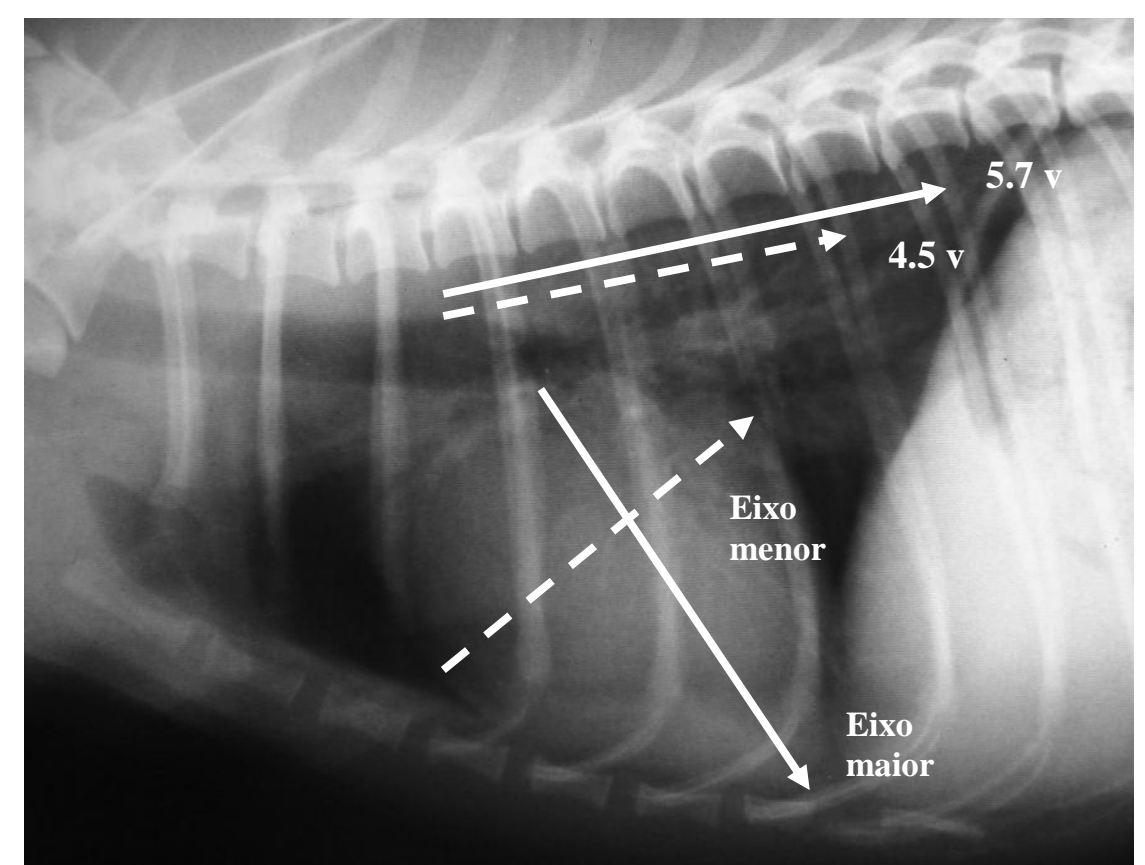

Figura 3. Radiografia lateral direita do tórax de cão normal da raça Yorkshire Terrier, mostrando o método de mensuração vertebral heart size (VHS). Esse animal apresenta VHS de 10.2 vértebras (v).

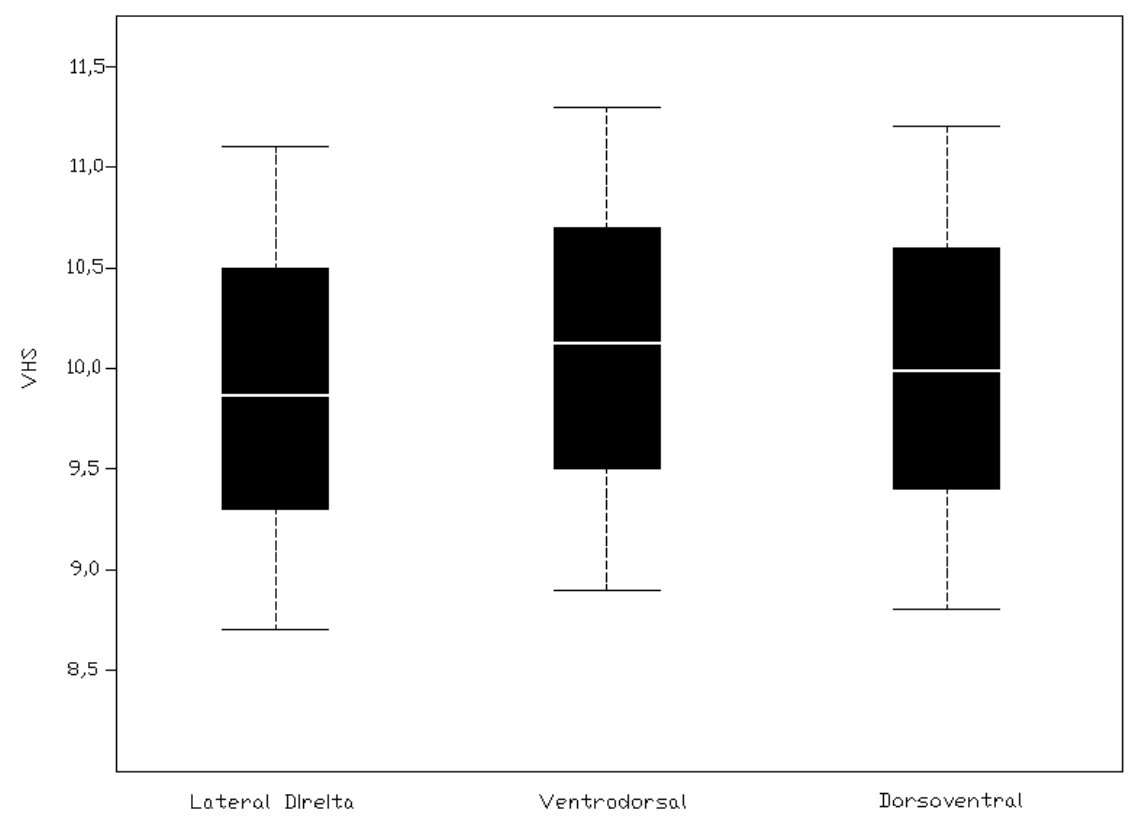

Figura 4. Valores do vertebral heart size (VHS) nas três projeções radiográficas realizadas em cães da raça Yorkshire Terrier. Observam-se as médias (linhas horizontais brancas), os desvios-padrão (retângulo preto) e os intervalos de confiança (entre as linhas horizontais pretas). 


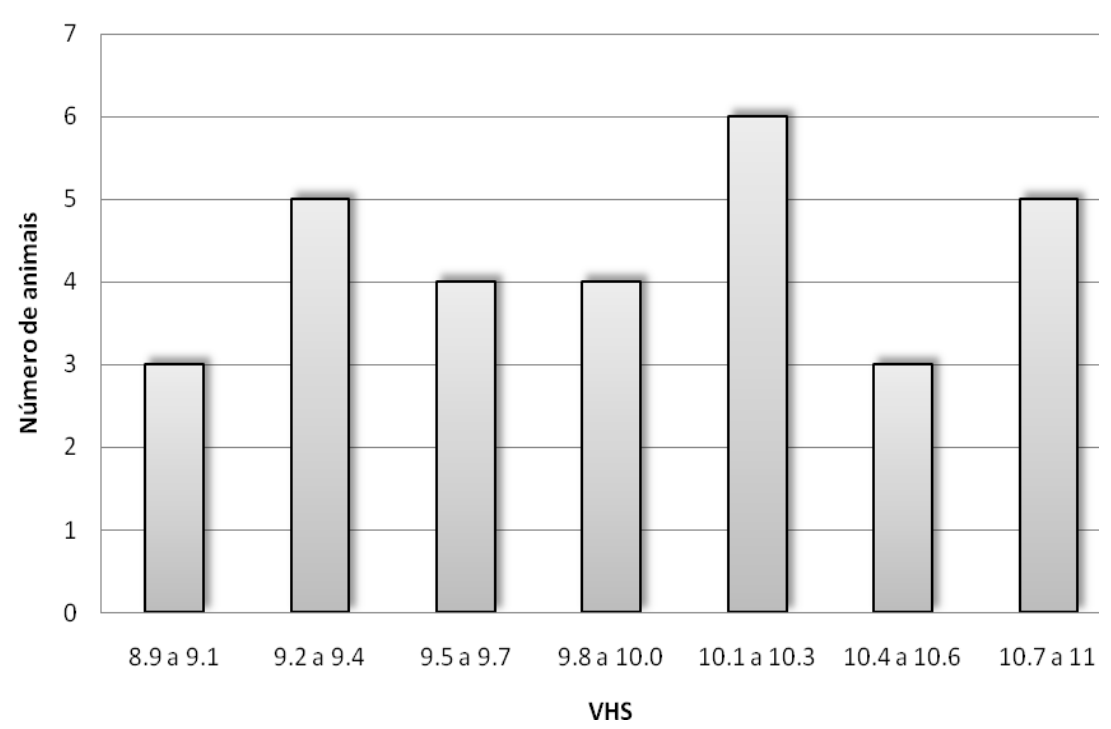

Figura 5. Distribuição dos valores do vertebral heart size (VHS) na projeção lateral de cães da raça Yorkshire Terrier clinicamente normais.

Na projeção ventrodorsal (VD), o valor médio de VHS foi de 10,1 $\pm 0,6$ vértebras, e o intervalo de confiança de 8,9 a 11,3 vértebras (Fig. 4). Na projeção dorsoventral (DV), o valor médio de VHS foi de $10.0 \pm 0.6$ vértebras, e o intervalo de confiança de 8,8 a 11,2 vértebras. O VHS na projeção VD não diferiu em relação à projeção DV (P>0,05). Estes resultados são semelhantes aos descritos por Buchanan e Bücheler (1995). Entretanto, ao contrário do que foi observado por esses autores, mas corroborando com Bavegems et al. (2005), o VHS nas projeções DV e VD não foram significativamente maiores que aqueles obtidos na projeção lateral $(\mathrm{P}>0,05)$. A conformação torácica de cães da raça Yorkshire Terrier pode explicar este resultado, pois em animais de tórax intermediário, como os deste estudo, a distância do coração ao filme é semelhante nas diferentes projeções radiográficas, resultando em menor distorção do contorno cardíaco. Isso representa maior aplicabilidade do método VHS em cães dessa raça.

Assim como observado por Buchanan e Bücheler (1995), Bavegems et al. (2005) e Marin et al. (2007), os valores de VHS não diferiram entre machos e fêmeas, em nenhuma das projeções radiográficas. Entretanto, ao contrário do que foi observado por Lamb et al. (2001), os valores de VHS apresentaram correlação positiva com a idade, porém com baixos coeficientes de correlação $-0.47 \leq \mathrm{r} \leq 0.50$ e $0.004 \leq \mathrm{P} \leq 0.007$-, em todas as projeções radiográficas. $\mathrm{O}$ peso corporal, por sua vez, após o cálculo dos coeficientes de Pearson, não se correlacionou com o VHS apenas na projeção dorsoventral $(\mathrm{r}=0,29$ e $\mathrm{P}=0,11)$, enquanto na projeção lateral direita e ventrodorsal a correlação foi baixa, mas significativa $(0,40 \leq \mathrm{r} \leq 0,42$ e $0,010 \leq \mathrm{P} \leq 0,027)$. Poucos autores avaliaram a correlação entre VHS e peso corporal. Basile (2008), ao estudar cães da raça Bulldog Inglês, não observou correlação significativa entre essas variáveis em nenhuma das projeções radiográficas, diferentemente do que foi encontrado neste trabalho, no qual se estudou uma amostra homogênea em peso corporal e idade. Os valores de VHS médio, desvio-padrão e correlações com peso corporal e idade em todas as projeções radiográficas estudadas estão sumarizados na Tab. 1.

A razão da profundidade com a largura torácica (Pt:Lt) apresentou valor médio de 0,75 $\pm 0,06$, sendo 0,66 o mínimo e 0,89 o máximo encontrado. De acordo com a qualidade do tórax, $53 \%$ dos animais apresentaram tórax largo, e $47 \%$ tórax intermediário. Não foi observada correlação entre a qualidade do tórax e o VHS ($0,009 \leq \mathrm{r} \leq 0,124$ e $\quad 0,51 \leq \mathrm{P} \leq 96$ ), como foi observado por Buchanan e Bücheler (1995) e Basile (2008). 
Tabela 1. Valores médios do vertebral heart size (VHS) e desvios-padrão, nas três projeções radiográficas, de cães da raça Yorkshire Terrier, e suas correlações com peso corporal e idade

\begin{tabular}{cccc}
\hline \multicolumn{1}{c}{ Variável ${ }^{(1)}$} & Média \pm desvio-padrão & Correlação com peso corporal & Correlação com idade \\
& & $\mathrm{r}$ & $\mathrm{R}$ \\
\hline VHS - LL (v) & $9,9 \pm 0,6$ & 0,42 & 0,47 \\
VHS - VD (v) & $10,1 \pm 0,6$ & 0,40 & 0,50 \\
VHS - DV (v) & $10,0 \pm 0,6$ & $\mathrm{~ns}$ & 0,50 \\
\hline
\end{tabular}

(1) Variável: VHS - LL = valor do VHS mensurado na projeção lateral direita; VHS - VD = valor de VHS mensurado na projeção ventrodorsal; VHS $-\mathrm{DV}=$ valor de VHS mensurado na projeção dorsoventral; $\mathrm{v}=$ vértebras.

(2) (3) Considerada significativa quando o coeficiente de correlação de Pearson (r) $\geq 0,40$ e $P<0,05$. Nos casos em que $r$ se aproximou de zero, a correlação foi considerada não significativa (ns).

Obs.: Não houve diferença significativa entre sexos.

\section{CONCLUSÕES}

Diante das condições em que foi realizada esta pesquisa e de acordo com os resultados obtidos em cães da raça Yorkshire Terrier, pode-se concluir que os valores de VHS devem ser correlacionados com raça, peso corporal e idade, assim como projeção radiográfica, uma vez que podem ser influenciados por esses fatores, permitindo, assim, uma avaliação cardíaca mais fidedigna. Além disso, em projeções laterais, o valor médio de VHS é de 9,9 90,6 vértebras, e o limite superior sugerido para cães da raça Yorkshire Terrier, em razão de profundidade:largura do tórax de $0,75 \pm 0,06$, é de 11,0 vértebras.

\section{REFERÊNCIAS BIBLIOGRÁFICAS}

BASILE, A.L.C. Avaliações ecodopplercardiográfica, eletrocardiográfica computadorizada, radiográfica e morfométrica em cães adultos da raça Bulldog Inglês. 2008. 103f. Dissertação (Mestrado em Medicina Veterinária) - Escola de Veterinária, Universidade Federal de Goiás, Goiânia.

BAVEGEMS, V.; CAELENBERG, A.V.; DUCHATEAU, L. et al. Vertebral heart size ranges specific for Whippets. Vet. Rad. Ultrasound, v.46, p.400-403, 2005.

BUCHANAN, J.W.; BÜCHELER, J. Vertebral scale system to measure canine heart size in radiographs. J. Am. Vet. Med. Assoc., v.206, p.194-199, 1995.

CASTRO, M.G.; VEADO, J.C.C.; SILVA, E.F. et al. Estudo retrospectivo ecodopplercardiográfico das principais cardiopatias diagnosticadas em cães. Arq. Bras. Med. Vet. Zootec., v.61, p.12381241, 2009.
FERIAN, P.E. Avaliação histológica, histoquímica, morfométrica, e radiográfica de traqueias de cães portadores de colapso traqueal. 2009. 100f. Tese (Doutorado em Medicina Veterinária) - Escola de Veterinária, Universidade Federal de Minas Gerais, Belo Horizonte.

GHADIRI, A.; AVIZEH, R.; RASEKH, A. Radiographic measurement of vertebral heart size in healthy stray cats. J. Feline Med. Surg., v.10, p.61-65, 2008.

HANSSON, K. Diagnostic imaging of cardiopulmonary structures in normal dogs and dogs with mitral regurgitation. 2004. 46f. Doctoral Thesis - Swedish University of Agricultural Sciences, Uppsala.

KEALY, J.K.; McALLISTER, H. Radiologia $e$ Ultrassonografia do Cão e do Gato. 3. ed. São Paulo: Manole, 2005. 436p.

LAMB, C.R.; WIKELEY, H.; BOSWOOD, A. et $a l$. Use of breed-specific ranges for vertebral heart scale in $\operatorname{dogs}$ as an aid to radiographic diagnosis of cardiac disease. Vet. Record, v.148, p.707-711, 2001.

LAMB, C.R.; BOSWOOD, A. Role of survey radiography in diagnosing canine cardiac disease. Comp. Cont. Ed. Pract. Vet., v.24, p.316-326, 2002.

LITSTER, A.L.; BUCHANAN, J.W. Vertebral scale system to measure heart size in radiographs of cats. J. Am. Vet. Med. Assoc., v.216, p.210214, 2000.

MARIN, L.M.; BROWN, J.; McBRIEN, C. et al. Vertebral heart size in retired racing Greyhounds. Vet. Rad. Ultrasound, v.48, p.332334, 2007. 
MAROLF, A.; BLAIK, M..; SPECHT, A. A retrospective study of the relationship between tracheal collapse and bronchiectasis in dogs. Vet. Rad. Ultrasound, v.48, p.199-203, 2007.

McREADY, D.M.; JOHNSON, L.R.; POLLARD, R.E. Fluoroscopic and radiographic evaluation of tracheal collapse in dogs; 62 cases (2001 2006). J. Am. Vet. Med. Assoc., v.230, p.8701876, 2007.

NAKAYAMA, H.; NAKAYAMA, T.; HAMLIM, R.L. Correlation of cardiac enlargement as assessed by vertebral heart size and echocardiographic and electrocardiographic findings in dogs with evolving cardiomegaly due to rapid ventricular pacing. J. Vet. Inter. Med., v.15, p.217-221, 2001.
PINTO, A.C.B.C.F.; IWASAKI, M. Avaliação radiográfica da silhueta cardíaca pelo método de mensuração VHS (vertebral heart size) em cães da raça Poodle clinicamente normais. Braz. J. Vet. Res. Anim. Sci., v.41, p.261-267, 2004.

SAMPAIO, I.B.M. Estatística Aplicada à Experimentação Animal. 2.ed. Belo Horizonte: FEPMVZ, 2002. 265p.

SOARES, E.C.; LARSSON, M.H.M.A.; PINTO, A.C.B.C.F. Aspectos radiográficos da doença valvar crônica. Cienc. Rural, v.34, p.119-124, 2004. 